\title{
Neue Erkenntnisse vom SABCS 2008
}

\author{
Gesprächsleitung: Raimund Jakesz ${ }^{\mathrm{a}}$ \\ Teilnehmer: \\ Peter Dubsky ${ }^{\mathrm{a}}$ Holger Eidtmann ${ }^{c}$ \\ Christian Schem ${ }^{c} \quad$ Alois Lang ${ }^{b}$ \\ Beat Thürlimann ${ }^{\mathrm{d}}$
}

${ }^{a}$ Klinische Abteilung für Allgemeinchirurgie, Medizinische Universität Wien,

${ }^{\mathrm{b}}$ Innere Medizin, LKH Feldkirch, Österreich

c Universitäts-Frauenklinik Kiel, Deutschland

${ }^{\mathrm{d}}$ Brustzentrum, Kantonsspital St. Gallen, Schweiz

Frage 1: Wie haben die Ergebnisse der Sequenzanalysen von BIG 1-98 und ABCSG-08 Ihre Einschätzung der endokrinen Adjuvanstherapie beeinflusst?

Dubsky: Aus der vorhandenen Information sind aus heutiger Sicht nur zwei wesentliche Schlussfolgerungen möglich:

I. Die absoluten Unterschiede hinsichtlich des krankheitsfreien Überlebens (DFS) sind zwischen einer Sequenz- (in welcher Reihenfolge auch immer) und einer Aromatasehemmer (AI)-«Upfront»-Therapie sehr moderat (wenn auch zum Teil statistisch signifikant). Die Ergebnisse sollten vor einer weiteren akademischen Analyse und nach entsprechendem Peer-Review als volles Manuskript publiziert werden. Die Präsentationen waren hinsichtlich der gewählten Endpunkte nicht eindeutig vergleichbar, und auch innerhalb der einzelnen Präsentationen bleibt eine Reihe von Fragen nicht zuletzt aufgrund der Zeitbegrenzung unbeantwortet. Ein Beispiel: Die Hazard Ratios (HR) hinsichtlich DFS der BIG 1-98-Monotherapie-Analyse haben sich im Vergleich zur letzten Analyse (medianes Follow-Up 51 Monate) eher verschlechtert (vormals 0.82 nun 0.88 ; «intention to treat»-Analyse). Gleichzeitig findet sich eine Verbesserung der HR hinsichtlich des Gesamtüberlebens (vormals 0.91 nun 0.87). Eine Aufschlüsselung der Ereignisse (brustspezifisch vs. andere Ursachen) wird hier notwendig werden, um die Ergebnisse nachvollziehbar zu machen.

II. Die ersten 5 Jahre endokrine Adjuvanstherapie sollten zumindest 2 Jahre Therapie mit einem AI beinhalten. Jede weitere Richtlinie ist zu diesem Zeitpunkt von rein akademischem Interesse, da hier die individuelle Patientin und der Behandlungsrahmen (z.B. innerhalb oder außerhalb von Studien, Remuneration durch Krankenkassen etc.) vermutlich den größeren Ausschlag geben werden. Im Kampf um statistisch signifikante Ergebnisse ist ein wenig die Anwendung in unserer klinischen Praxis untergegangen. Ein Beispiel: Der absolute DFS-Unterschied zwischen Letrozol alleine (87.9\%) und der Sequenz Tamoxifen-Letrozol (86.2\%) liegt nach 5 Jahren bei $1.7 \%$. Wie relevant kann dieser Unterschied sein, wenn weitere, typische Informationen über die Patientin vorliegen (z.B. die Verträglichkeit von Letrozol ist sehr schlecht oder sehr gut, Tamoxifen hatte unerträgliche Wallungen zur Folge, die Patientin nimmt Serotonin-Wiederaufnahme-Hemmer (SSRI) oder es liegt eine klinisch manifeste Osteoporose vor)? Neben dieser sinnvollen Individualisierung aufgrund sehr schlichter, klinischer Beobachtungen ist auch die individuelle Pharmakokinetik von Tamoxifen ein zukunftsreiches Thema. Eine individuelle Anpassung nach CYP2D6-Genotyp muss validiert werden, erscheint aber aufgrund der von Goetz et al. präsentierten Daten aussichtsreich. Vor allem ist derzeit unklar, inwiefern der CYP2D6-Genotyp eine Rolle spielt, wenn 2 Jahre AI-Therapie in den ersten 5 Jahren endokriner Therapie inkludiert sind.

Eidtmann/Schem: Unsere bisherige Einschätzung der adjuvanten endokrinen Therapie postmenopausaler Patientinnen mit Mammakarzinom folgte unter anderem zwei Leitsätzen: 1. AI sind Tamoxifen überlegen. 2. Man sollte mit dem effektivsten Therapeutikum beginnen. Insofern haben wir immer die Upfront-AI-Therapie favorisiert. Durch die genannten Studien fühlen wir uns in unserer bisherigen Einschätzung bestätigt. Die Sequenz Tamoxifen gefolgt von Letrozol scheint nach den BIG 1-98-Daten zumindest hinsichtlich der Brustkrebsereignisse unterlegen zu sein, auch wenn Vorsicht geboten ist, da keine statistischen Signifikanzen angegeben sind. Die ABCSG-08 Daten bestätigen, das Anastrozol Tamoxifen überlegen ist, auch nach 2-jähriger Vorbehandlung mit Tamoxifen.

Lang: Die Upfront-AI-Therapie ist zu bevorzugen gegenüber Switching oder sequentiellen Konzepten, insbesondere wegen

\section{KARGER}

Fax +497614520714

Information@Karger.de

www.karger.com (c) 2009 S. Karger GmbH, Freiburg

Accessible online at:

www.karger.com/brc
Prof. Dr. med. Raimund Jakesz

Klinische Abteilung für Allgemeinchirurgie

Medizinische Universität Wien

Währinger Gürtel 18-20, 1090 Wien

Tel. +43 1 40400-6916, Fax -6918

raimund.jakesz@meduniwien.ac.at 
dem in den ersten 2 Jahren auftretenden Peak an Rezidiven unter Tamoxifen. Das Fehlen eines Overall-Survival-Benefits für die Upfront-Therapie ist störend.

Thürlimann: Die Sequenzanalysen der BIG 1-98-Studie zeigen - entgegen den Erwartungen der meisten Kollegen - keinen Vorteil einer Sequenz von Tamoxifen und Letrozol gegenüber einer 5-jährigen Therapie mit Letrozol alleine. Ich persönlich habe zur Patientenbetreuung nie die Resultate der Switch-Strategie auf die Sequenzstrategie extrapoliert. Darum war für mich persönlich die fehlende Überraschung eben keine Überraschung.

\section{Frage 2: Das Fehlen eines Overall-Survial-Benefits für die Upfront-Therapie steht im Gegensatz zu den Ergebnissen der Sequenz- und Switching-Studien. Wo sehen Sie die Indikation für einen sequentiellen Ansatz?}

Dubsky: BIG 1-98 liefert nun den erkenntnistheoretischen Vorteil, dass drei Therapieansätze, welche einen AI beinhalten, innerhalb einer einzelnen Studie verglichen werden können. Die genaue Aufarbeitung dieser Daten wird zeigen, ob hier tatsächlich relevante Unterschiede bestehen. Aus heutiger Sicht erscheinen die Unterschiede zwischen Upfront und Sequenz sehr moderat (wenn nicht minimal) und eine Veränderung der derzeitigen Richtlinien (z.B. St. Gallen) ist nicht angemessen.

Eidtmann/Schem: Für die Sequenz Tamoxifen $\rightarrow$ AI sehen wir eine klare Indikation bei perimenopausalen Patientinnen, wo sich aufgrund des endokrinen Status der Patientin eine Upfront-Therapie verbietet. Nach 2-3 Jahren Tamoxifen sollte nach Sicherung des postmenopausalen Status auf einen AI umgestellt werden. Nach den vorgestellten BIG 1-98-Daten ist die Sequenz Letrozol $\rightarrow$ Tamoxifen der alleinigen Therapie mit dem AI nicht unterlegen. Man wird also insbesondere bei eingeschränkter Verträglichkeit eines AI nach 2 Jahren den Wechsel auf Tamoxifen erwägen können.

Lang: Siehe meine Antwort zu Frage 1.

Thürlimann: Die Intention-to-treat-Analyse der BIG 1-98Studie zeigt einen recht klaren Trend zu einem Gesamtüberlebensgewinn. Werden die auf den Tamoxifenarm randomisierten Patientinnen, welche nach Entblindung des Tamoxifenarms selektiv die Tamoxifen-Behandlung gestoppt und stattdessen Letrozol eingenommen haben, zum Zeitpunkt der Beendigung der Tamoxifen-Therapie zensuriert, besteht ein klarer Gesamtüberlebensvorteil zugunsten von Letrozol. Diese aus ethischen Gründen durchgeführte Entblindung erschwert die Analyse und Interpretation der Ergebnisse. Vermutlich liegt die Verbesserung des Gesamtüberlebens durch
Letrozol - verglichen mit Tamoxifen - zwischen den Werten, die wir durch die Intention-to-treat- (13\%) und die zensurierte Analyse (19\%) erhalten. Die IBCSG ist daran, ein entsprechendes statistisches Modell zu erarbeiten, um besser zu verstehen, welche Faktoren zu berücksichtigen sind, um die durch das selektive Crossover entstandene Situation besser $\mathrm{zu}$ analysieren. Eine Indikation für den sequentiellen Ansatz sehe ich dort, wo Kontraindikationen und Nebenwirkungen einen Wechsel von Letrozol auf Tamoxifen wünschenswert erscheinen lassen.

\section{Frage 3: Welchen Stellenwert hat die 5-jährige Behandlung mit Tamoxifen? Gibt es noch einen Platz dafür?}

Dubsky: Die Indikation für Tamoxifen alleine (bei der postmenopausalen Patientin mit invasivem Karzinom) nimmt immer weniger Raum ein. Die relative Risikoreduktion durch einen AI ist bei genauer statistischer Analyse (z.B. durch Subgroup Treatment Effect Pattern Plot; STEPP) für alle Subgruppen ähnlich. Damit sollten weder ältere, nodal negative Patientinnen, noch Patientinnen mit bestimmten HormonrezeptorKonstellationen von vornherein mit Tamoxifen alleine behandelt werden. Allerdings ergibt die relative Risikoreduktion bei Patientinnen mit sehr guter Prognose nur wenig absoluten Gewinn. Daraus ergibt sich ein therapeutischer Spielraum, welcher vor allem bei Verträglichkeitsproblemen oder im Einzelfall auch bei Osteoporose zum Einsatz kommen kann.

Eidtmann/Schem: Die 5-jährige Tamoxifentherapie (allein oder in Kombination mit einem LHRH-Analogon) bleibt Standard der adjuvanten endokrinen Therapie der prämenopausalen Patientin mit Brustkrebs. In der Postmenopause sehen wir die Indikation eingeschränkt auf Patientinnen mit massiven Beschwerden unter einem AI, bei denen die Alternative ansonsten der Abbruch der adjuvanten endokrinen Therapie wäre. Und natürlich bleibt die 5-jährige Tamoxifentherapie eine Option in der Prävention und beim Hormonrezepor-positiven DCIS.

Lang: Die Gewichtung von zu erwartenden Nebenwirkungen und vor allem die Pharmakogenetik von Tamoxifen (poor, intermediate und extensive Metabolizer) sind zu bestimmen. Die poor Metabolizer sind von einer Tamoxifen-Therapie auszuschließen. Als Beispiele für eine patientenbezogene, individualisierte Therapie außerhalb von Studien sind Patientinnen mit einer Anamnese an thrombembolischen Ereignissen (Trägerinnen eines Faktor-V-Leidens) bevorzugt mit AI zu behandeln, Patientinnen mit Osteoporose nach Bestimmung des Metabolisierungstyps bevorzugt mit Tamoxifen.

Thürlimann: Für Patientinnen mit Kontraindikationen oder Unterverträglichkeit von AI sowie für Patientinnen mit sehr
56

Breast Care 2009;4:55-60
Jakesz/Dubsky/Eidtmann/Schem/Lang/ Thürlimann 
kleinem Rückfallrisiko ist die Behandlung mit Tamoxifen eine durchaus vertretbare Behandlungsoption. Vor- und Nachteile sowie die verschiedenen Verträglichkeitsprofile sollten mit der Patientin besprochen werden.

Frage 4: Welche Patientinnen, die für eine TamoxifenTherapie vorgesehen sind, sollten auf ihren CYP2D6Metabolismus getestet werden? Ist es für alle sinnvoll?

Eidtmann/Schem: Das Enzym Cytochrom P450 2D6 (CYP2D6) katalysiert die Aktivierung des Tamoxifens zu Endoxifen, einem wesentlichen aktiven Metaboliten. Die metabolische Aktivität von CYP2D6 ist von äußeren, aber auch von genetischen Faktoren abhängig. So ist von bestimmten Polymorphismen bekannt, dass sie mit einer geringeren Aktivität assoziiert sind. Diese poor Metabolizer zeigten in einigen retrospektiven Untersuchungen ein schlechteres Ansprechen auf Tamoxifen, in anderen nicht. Nachdem Goetz et al. bereits in einem Subkollektiv der NCCTG 89-30-52 Studie ein schlechteres rezidivfreies Überleben der poor Metabolizer unter Tamoxifen zeigen konnten, gelang ihnen dies jetzt auch in einem Subkollektiv der ABCSG-08 Studie. Die Ergebnisse sind vielversprechend, aber noch nicht reif für die Routine: Es gibt widersprüchliche Ergebnisse anderer Autoren und es fehlen prospektive Untersuchungen.

Dubsky: Die von Goetz und Kollegen präsentierten Ergebnisse konnten nur für Frauen mit 5-jähriger Tamoxifen-Therapie ein signifikant erhöhtes Risiko der poor Metabolizer feststellen. Jene Patientinnen mit schlechter Tamoxifen-Metabolisierung, welche in der Sequenz Anastrozol laut Studienprotokoll erhielten (s. ABCSG-08) zeigten kein erhöhtes Risiko. Damit kann zumindest aus diesen Daten heraus keine Empfehlung für «Sequenz-Patientinnen» gemacht werden. Beispielhaft ist, dass hier das Daten- und Präparatmaterial aus einer gut validierten, prospektiv randomisierten Studie verwendet wurde. Dies erhöht den Wert der Analyse gegenüber einer Reihe von ähnlichen Untersuchungen, welche auf retrospektiv erhobenen Daten beruhen. Es ist zu erhoffen, dass die gezeigten Ergebnisse anhand ähnlich prominenter Datensätze validiert werden können.

Lang: Ganz klar: Alle Patientinnen, bei denen eine Tamoxifen-Therapie vorgesehen ist, sollten einer Testung des CYP2D6-Metabolismus unterzogen werden. $\mathrm{Zu}$ berücksichtigen sind auch die Interferenzen mit SSRI und mit Opioiden.

Thürlimann: Wir führen routinemäßig keine solchen Tests durch und warten auf die entsprechenden Resultate der translationalen Studie der BIG 1-98, welche in mehreren Labors durchgeführt und durch die IBCSG-Statistiker ausgewertet wird.

Neue Erkenntnisse vom SABCS 2008

\section{Frage 5: Welchen Wert sehen Sie in der klinischen Praxis für die Bestimmung von Mikrometastasen im Knochenmark oder in der Peripherie?}

Dubsky: Der Wert für die klinische Praxis ist nach wie vor marginal. Im adjuvanten Bereich, jedoch auch in der Therapie des metastasierten Mammakarzinoms findet sich nur ein geringfügiger Prozentsatz von Patientinnen, welche tatsächlich gut reproduzierbare Mikrometastasen aufweisen. Weiterhin scheinen die Nachweismethoden (sowohl aus Knochenmark wie auch aus Blut) außerhalb der hoch spezialisierten Laboratorien nur wenig reproduzierbar zu sein. Die geringe Frequenz und die derzeit vorhandenen Methoden machen einen Einsatz im Klinikalltag sehr schwierig.

Eidtmann/Schem: Wissenschaftlich ist der prognostische Wert unserer Meinung nach unumstritten. Trotzdem konnten die Methoden zum Nachweis von Mikrometastasen im Knochenmark ihre Praxistauglichkeit bisher nicht beweisen. Dies mag sich jetzt ändern durch den automatisierten Nachweis zirkulierenden Tumorzellen im peripheren Blut.

Lang: Die Bestimmung von Mikrometastasen hat in der klinischen Routine keinen Platz, sehr wohl aber in Studien.

Thürlimann: Tatsächlich hat die vermehrte Standardisierung und Validierung dieser Maßnahmen neues Interesse an diesen Parametern geweckt. Allerdings wird der praktische Wert dadurch etwas eingeschränkt, dass die Strategie zur Behandlung des Mammakarzinoms seit längerem vom primär risikoadaptierten Vorgehen zu «first: select the target» gewechselt hat. Der prädiktive Wert dieser Untersuchungen für eine bestimmte adjuvante therapeutische Intervention scheint mir noch nicht genügend etabliert.

\section{Frage 6: Welchen zusätzlichen Wert hat lhrer Meinung nach die Bestimmung der HER2-Protein-Expression? Sollte sie Eingang in die klinische Praxis finden?}

Dubsky: A. Lipton präsentierte hier sehr beeindruckende Daten. Dabei zeige sich, dass gut $13 \%$ der Patientinnen mit einer HER2-Genamplifikation unter der gewählten Proteinexpressionsschwelle des «HERmark assays» lagen. Genau diese Patientinnen waren deutlich unterlegen hinsichtlich ihres progressionsfreien Überlebens. Die Daten sollten dringend anhand von prospektiv erstellten Datensätzen validiert werden. Ein Eingang in die Klinik ist derzeit noch nicht abzuschätzen.

Eidtmann/Schem: Neben den etablierten Bestimmungen des HER2-Status mittels Immunhistochemie oder FISH-Testung werden weiter Modifikationen und Alternativen untersucht. So wurde in San Antonio ein quantitatives Verfahren zur Be- 
stimmung des totalen sowie homodimerisierten HER2-Proteins vorgestellt. Retrospektiv wurde die prädiktive Bedeutung für das Ansprechen auf eine Trastuzumab-Therapie an einen Kollektiv von HER2-FISH-positiven Patientinnen eines Zentrums untersucht. Es ist aber sicherlich verfrüht, die ersten so gewonnenen positiven Ergebnisse für die klinische Routine zu empfehlen.

Lang: Die Bestimmung der HER2-Expression sollte um den «truncated» HER2-Rezeptor erweitert werden, da bei dieser Situation Herceptin deutlich weniger wirksam bis unwirksam ist.

Thürlimann: Die routinemäßige Bestimmung des HER2-Status mit standardisierten und validierten Testkits, speziell ausgebildetem Personal und externer Qualitätskontrolle sollte zum Standard gehören, um eine entsprechende zielgerichtete Therapie durchführen zu können.

\section{Frage 7: Die Kombination von zielgerichteter Therapie und endokriner Intervention war Gegenstand zahlreicher in San Antonio gezeigter Forschungs- ergebnisse. Welche zytostatikafreien Kombinationen sind «Ready for Prime Time»?}

Dubsky: Prime Time adjuvante Therapie? Nein, gar nicht. - Prime Time palliative Therapie? Nicht Prime Time, aber in vielen Situation in denen vor allem endokrine Therapie alleine wenig Erfolg versprechend erscheint und eine Chemotherapie nicht möglich ist, könnten einzelne Kombinationen eingesetzt werden. Beispielhaft sind hier die TAnDEM-Studie (Anastrozol+/-Trastuzumab) und der EGF30008 Trial (Letrozol+/- Lapatinib). Beide Studien zeigten deutliche Erfolge hinsichtlich des progressionsfreien Überlebens bei der Erstlinientherapie in der metastasierten Situation. Es sei kritisch hinzugefügt, dass beide Ergebnisse nur auf Patientinnen mit Überexpression von HER2 angewendet werden können. Auch haben diese Studien (dem Setting völlig angemessen) keinen Chemotherapie-Zweig. Wie so oft: Außerhalb von Studienprotokollen ist keine eindeutige Empfehlung auszusprechen.

Eidtmann/Schem: Die Kombintion von AI und Anti-HER2Therapie hat in Phase-III-Studien ein verbessertes progressionsfreies Überleben beim HER2-positivem Mammakarzinom gegenüber einer alleinigen AI-Therapie zeigen können. Schon länger, nämlich aus der TAnDEM-Studie, war dies für die Kombination Anastrozol/Trastuzumab bekannt. In San Antonio wurde jetzt die EGF3008-Studie mit der Kombination Letrozol/Lapatinib beim Hormonrezeptor- und HER2positiven, metastasierten Mammakarzinom vorgestellt. Die Kombination zeigte eine Verbesserung des medianen progressionsfreien Überlebens von 3 auf 8 Monate. Beide Kombinationen sind aus unserer Sicht in der Routine einsetzbar.
Lang: Die Aufschlüsselung und dementsprechend zielgerichtete Therapie der einzelnen Subklassen von Brustkrebs ist weiter $\mathrm{zu}$ forcieren. Patientinnen mit dreifach positivem $(\mathrm{ER}+, \mathrm{PR}+\mathrm{HER} 2+)$ und nur HER2-positvem Brustkrebs werden weitgehend gleich behandelt, es handelt sich jedoch sehr wohl unterschiedliche Entitäten. «Ready for Prime Time» sind zytostatikafreie Kombinationen vor allem bei der älteren Frau, hier ist aber ein Mangel an Untersuchungen zu konstatieren.

Thürlimann: In der adjuvanten Situation sehe ich keine solchen Kombinationstherapien als etabliert. In der fortgeschrittenen Situation besteht kaum ein Zweifel, dass Kombinationstherapien das progressionsfreie Überleben verlängern können. Allerdings bleibt es unklar, ob dies mit einer Sequenztherapie nicht ebenfalls möglich wäre. Zudem ist unklar, wie diese zytostatikafreien Kombinationen im Gesamtarmamentarium der palliativen Möglichkeiten am besten eingesetzt werden sollen.

\section{Frage 8: Welche Therapieansätze halten Sie für aus- sichtsreich beim dreifach negativen Mammakarzinom?}

Dubsky: Es ist hier wenig sinnvoll «Tipps» abzugeben. Das dreifach negative Karzinom ist neben der HER2 überexprimierenden Subgruppe vermutlich die homogenste Brustkrebs-Subgruppe. Tatsächlich stimmen hier Phänotyp und die «basal type» molekulargenetische Signatur in einer deutlichen Mehrheit der Fälle überein. Gleichzeitig haben diese Patientinnen vor allem innerhalb der ersten 5 Jahre ein enorm hohes Rezidivrisiko. Das dreifach negative Karzinom hat vor allem in den neoadjuvanten Chemotherapieansätzen eine hohe Sensibilität gezeigt. Derzeit wird eine Reihe von «Biologicals» in chemotherapeutische Kombinationen mit einbezogen. Der wesentlichste zu empfehlende Therapieansatz ist derzeit vermutlich die Behandlung der Patientinnen innerhalb eines Studienprotokolls.

Eidtmann/Schem: Die Forschung geht eindeutig in Richtung der Kombination von Zytostatika mit zielgerichteten Therapieformen. Wir erwarten vor allem hochgradige synergistische Effekte aus der Kombination von platinhaltiger Chemotherapie mit den sogenannten PARP-Inhibitoren.

Lang: Studien mit Kombinationen von Cytostatika, speziell Cisplatin und Analoga in Kombination mit EGFR-Antikörpern (z.B. BALI) sind ein Weg, die «small Molecules» sind zu prüfen.

Thürlimann: Die dreifach negative Population ist biologisch eine heterogene Population und muss wohl weiter unterteilt werden, um zielorientierte Therapien durchführen zu können. In letzter Zeit haben vor allem DNA-schädigende Substan- 
zen wie Alkylantien und Platin bei der BRCA1-Mutation des Tumors, sowie PARP-Inhibitoren das Interesse der Forscher und Kliniker geweckt. Diese Substanzen sollten als erste systematisch in dieser Patientenpopulation untersucht werden.

\section{Frage 9: Antrazykline und Taxane, Sequenz oder Kombination - welche neuen Erkenntnisse gab es in San Antonio?}

Dubsky: Neue Erkenntnisse sollten eher nach der Publikation und dem Peer Review gewonnen werden. Aus den kurzen Präsentationen ist der Wissensgewinn eher mager. In der NSABP 30-Studie konnte anhand von 5351 Patientinnen gezeigt werden, dass 24 Wochen Therapie nach dem ACT-Schema nur marginal besser sind als 12 Wochen Therapie nach dem TAC- oder AT-Schema. Dieser Eindruck wird durch die BCIRG005-Daten bestätigt. Bei ähnlicher Therapiedauer gleichen sich die Effektivitäten der Schemata wieder an. - Ein fast déjà-vu-artiges Erlebnis, nachdem im Rahmen des SABCS 2007 die TACT-Studie präsentiert wurde.

Eidtmann/Schem: Es wurden drei große Studien zur adjuvanten Therapie mit Anthrazyklinen und Taxotere (T) vorgestellt: die NSABP B-30-, die BCIRG 005- und die deutsche EC-Doc-Intergroup-Studie. Die NSABP untersuchte dreiarmig $4 \times \mathrm{AC} \rightarrow 4 \times \mathrm{T}, 4 \times \mathrm{AT}$ und $4 \times \mathrm{TAC}$ mit dem Fazit, dass die Sequenz den Kombinationen überlegen ist, und zwar deutlich gegenüber $4 \times$ AT und marginal gegenüber $4 \times$ TAC. Die BCIRG fand keinen Unterschied in der Effektivität von $4 \times \mathrm{AC} \rightarrow 4 \times \mathrm{T}$ versus $6 \times \mathrm{TAC}$ aber ein unterschiedliches Toxizitätsprofil mit ausgeprägter Hämatotoxizität bei TAC und nicht-hämatologischer Toxizität bei der Sequenz. In der EC-Doc-Studie wurden bei 1-3 befallenen Lymphknoten $6 \times \mathrm{FE}(100) \mathrm{C}$ verglichen mit $4 \times \mathrm{EC} \rightarrow 4 \times \mathrm{T}$ mit Überlegenheit der Sequenz hinsichtlich ereignisfreiem und Gesamtüberleben. Zusammenfassend scheinen $6 \times \mathrm{TAC}$ und $4 \times \mathrm{AC} \rightarrow 4 \times \mathrm{T}$ bzw. $4 \times \mathrm{EC} \rightarrow \mathrm{T}$ gleichwertig und möglich beim nodal positiven Mammakarzinom. Vom Nebenwirkungsprofil und der subjektiven Verträglichkeit tendieren wir eher zum TAC.

Lang: Die Sequenz von AC, gefolgt von Taxan hat in der NSABP 30 für das krankheitsfreie Überleben einen statistisch signifikanten Vorteil ergeben, diese große, dreiarmige Chemotherapie-Studie mit über 1700 Patientinnen ist zu berücksichtigen, auch die Toxizitätsdaten sprechen für die sequentielle Anwendung.

Thürlimann: Ich glaube nach wie vor, dass Taxane, falls sie in der adjuvanten Situation als angezeigt angesehen werden, am besten in Sequenz zu Antrazyklin gegeben werden.
Frage 10: Exemestan, Zoledronat, Tibolon, Denosumab - welche neuen Erkenntnisse gab es in San Antonio zur Knochengesundheit beim Mammakarzinom?

Dubsky: Die größte Enttäuschung war vermutlich, dass AZURE nicht präsentiert wurde. Gleichzeitig haben die ABCSG-12-Daten erneut Unterstützung durch die Überlebensdaten der ZO-Fast-Studie erhalten (auch in der Präsentation von ZO-FAST beim ESMO 2008). Der adjuvante Einsatz von Zoledronat abseits seiner Indikationen in Sachen Knochendichte bleibt damit abzuwarten. Eine ganze Reihe von Postern im Rahmen des SABCS machen einen ähnlichen Studienansatz (Studienziel: Überleben) für den Anti-RANKLigand-Antikörper Denosumab plausibel: a) Die Expression von RANK auf humanen und murinen Brustkarzinomen lässt sich nachweisen und b) eine Invasionshemmung/Migrationshemmung der Zelllinien durch Denosumab (bzw. durch RANK knockout) findet statt. c) Eine beeindruckende Abnahme von Brusttumoren durch RANKL-Inhibition im Mausmodell konnte gezeigt werden.

Eidtmann/Schem: Hinsichtlich Knochengesundheit und den genannten Substanzen gab es aus unserer Sicht nichts wesentlich Neues: Exemestan vermindert den Knochenmineralsalzgehalt wie die nicht-steroidalen AI. Tibolon erhöht zwar erwartungsgemäß die Knochendichte, ist aber nach Mammakarzinom obsolet. Zu Denosumab gab es wenig und nichts Neues. Wichtig sind die Ergebnisse aus unserer ZOFAST-Studie, nicht so sehr wegen der erwarteten günstigen Effekte auf die Knochendichte bei adjuvanter AI-Therapie, sondern wegen des günstigen Einflusses auf das rezidivfreie Überleben. Wie in der ABCSG-12-Studie bei prämenopausalen Patientinnen so fanden wir bei postmenopausalen Patientinnen eine signifikante Verbesserung des krankheitsfreien Überlebens bei halbjährlicher Infusion von $4 \mathrm{mg}$ Zoledronat mit einer ca. 40\%igen Risikoreduktion bezogen auf Lokalrezidive, Fernmetastasen und kontralaterale Karzinome.

Lang: Die gezielte Therapie für den Knochen mit Zoledronsäure und Denosumab ist im Vormarsch, leider haben wir keine guten prädiktiven Methoden, um die Patientinnen auszuwählen. Studien mit onkologischen Endpunkten wurden zu Zoledronsäure-Behandlung vorgestellt (ABCSG-12) und einige laufen noch.

Thürlimann: Knochengesundheit beim Mammakarzinom spielt eine zunehmende Rolle mit stärker eingreifenden, längeren Therapien sowie mit dem besseren Überleben der Patientinnen. Für Zoledronat wurden auf dem ASCO und in San Antonio Resultate und Updates von paradigmatischen Studien gezeigt. Zoledronat ist klar eine Behandlungsoption für Patientinnen in der Prämenopause, die eine kombinierte endokrine Therapie erhalten. Allerdings zeigen die Resultate aufgrund der speziellen Studiensituation noch nicht eine klare 
Indikation im Sinne eines Goldstandards an. Dazu benötigen wir weitere bestätigende Resultate aus großen Studien, welche wir bald erwarten. Denosumab zeigt - wie erwartet - erfolgversprechende Resultate und wird wohl bald den entsprechenden Platz in der Klinik einnehmen. Tibolon wird als Hormonersatztherapie häufig eingesetzt, wohl zu Recht. Allerdings unterscheidet es sich von den übrigen Hormonersatzpräparaten nicht grundsätzlich - entgegen den früheren Erwartungen und Vorschlägen.

\section{Teilnehmer}

Dr. med. Peter Dubsky

Klinische Abteilung für Allgemeinchirurgie

Medizinische Universität Wien

Währinger Gürtel 18-20, 1090 Wien, Österreich

Tel. +431 40400-5621, Fax -5641

peter.dubsky@meduniwien.ac.at

Dr. med. Holger Eidtmann/ Dr. med. Christian Schem

Universitäts-Frauenklinik

Haus 24, Arnold -Heller-Straße 3, 24105 Kiel

Tel.: +49 431 597-2099, Fax -2146

eidtmann@email.uni-kiel.de

Dr. med. Alois Lang

Innere Medizin

LKH Feldkirch

Carinagasse 47, 6807 Feldkirch, Österreich

Tel. +43 5522 303-2600

alois.lang@vol.at

Prof. Dr. med. Beat Thürlimann

Kantonsspital St. Gallen

Brustzentrum

9007 St. Gallen, Schweiz

Tel. +41 71 494-1177, Fax -6368

beat.thuerlimann@kssg.ch 